\title{
Incorporation of technology in health care: impact on costs
}

\author{
Seguridade Social — Instituto de Pesquisa \\ Econômica Aplicada
}

Health care costs reached US $\$ 1.7$ trillion in 1990. This is equivalent to $8 \%$ of the world's GNP. Governmental institutions spent US $\$ 1.0$ trillion, nearly $60 \%$ of the US $\$ 1.7$ trillion. Developing countries in Latin America, Asia and Africa spent US\$170 billion. That is the equivalent of $2 \%$ of the GNP for these countries.

In the early 1960s, the group of countries forming the OCDE spent an average of $3.8 \%$ of their respective GNPs. At the end of the 1980s these expenses reached $8 \%$ of the GNP.

A big share of the increase in expenditures can be explained by these factors:

1. Horizontal and vertical coverage extension.

Horizontal extension coverage means new segments of the population, for example, health insurance customers. This is a consequence of health insurance policies that made health care more available.Vertical coverage extension means the diversification of health care- and sanitary-related procedures in the gamut of universal protection.

2. Aging of the population. As it is well known, medical care to older individuals is more expensive.

3. Changes in the medical technology field.

Although spending more resources in health care is ethically defensible, several studies show that there is not a direct relationship between higher national expenditures in health care and an improvement in health conditions.

The question of how to monitor the increments in health expenses is critical for developing countries. In these countries, the acquisition of new and expensive technologies, done many times without sound analysis, consumes financial resources to privilege only a limited share of the population, whereas the great share of the population still requires primary health care.

The increase in medical costs placed technology in the center of the political discussions about how to cut health care costs. Studies show that technology, in a broad sense, is responsible for an average of 50\% increase in health care costs in recent years (3).

One single technological advancement, the Intensive Care Unit, generated an average increase of $10 \%$ in hospital costs (4). Banta and Thacker estimated the overall costs of electronic fetal monitoring in the U.S. for 1977-78. This technology is believed to be applied in 50\% of the women going into labor. Direct and indirect costs of electronic fetal monitoring reached US $\$ 411$ million. This amount of money is five times more than the amount spend in all private or public childrens' immunization programs (5).

Cost increase is caused not only by more expensive technologies on a unit basis (CT scan, organ transplants) but by new drugs and by small technologies (more simple laboratory procedures) that present higher additive costs.

It is also observed that the technologies for diagnostic purposes spread faster. These technologies are employed in varied clinical settings and on a growing number of people. Furthermore, they absorb a large number of skilled professionals and are in the majority of the cases additive technologies instead of substitutive technologies. The therapeutic technologies have a more restricted use except for drug prescription (6).

Spread of new technologies has been determined by the following factors:

- The tactical concept that any innovation is convenient.

- Innovations are a source of revenues for the manufacturers, and consequently, strong market pressure for their incorporation takes place.

- Sub-specialization and the use of technology as a strategy in maintaining the entries.

- The expansion of social protection systems, which bring into the medical market millions of subjects previously not covered.

- Reimbursement policies that foster the use of modern technologies.

- A consumer attitude from patients who rapidly learn information about new technologies.

In addition to assessing the technological innovations in terms of effectiveness, safety and social consequences within a context of limited resources, an assessment of costs related to effectiveness and usefulness is of great relevance. 
Ultimately, within this same context of limited resources, any decision regarding allocation of resources will mean deferring other requirements. When considering health care costs, the adoption of decisions not documented on the best cost against effectiveness ratio or the best cost/usefulness ratio will mean penalization of certain population groups.

It is very difficult to influence health care professionals, mainly those professionals who handle the leading technology, to consider the question of alternative use of resources. These professionals believe in the paradigm that all possible care should be dispensed to a given patient and that human life is precious.

The solution lies, perhaps, in establishing a system to assess technology applied to health care. This system should give more information either for political decisions in health care or individual decisions for professionals in the field.

The economics of health care has already obtained some instruments to assist in these decisions. On one side, the studies on effectiveness against costs allow the assessment of lower cost strategies to obtain a certain objective or the purchase of a technology in study with another reference technology. On the other side, analysis regarding cost against usefulness that allows the assessment of different alternatives in terms of costs, duration and quality of life.

The question of incorporation of technology in health care and its effect on costs is very complex. This complexity is due to interests and to the limited dissemination of the instruments for methodological assessments. However, due to the importance of the question as well as the strong participation of the state in financing health care, governments should promote mechanisms to make a continuous assessment possible. This would allow governments to play a more effective regulatory role in the incorporation and diffusion of technology in health care. 